\title{
Electroabsorption enhancement in disordered, strained InGaAs/GaAs quantum well
}

\author{
Joseph Micallef ${ }^{a)}$ \\ Department of Microelectronics, University of Malta, Msida MSD 06, Malta \\ E. Herbert Li ${ }^{\text {b) }}$ \\ Department of Electrical and Electronic Engineering, The University of Hong Kong, \\ Pokfulam Road, Hong Kong \\ Bernard L. Weiss ${ }^{\mathrm{C}}$ \\ Department of Electronic and Electrical Engineering, University of Surrey, \\ Guildford, Surrey GU2 5XH, United Kingdom
}

(Received 23 March 1995; accepted for publication 29 August 1995)

\begin{abstract}
The results of modeling the application of an external electric field to disordered, strained InGaAs/ GaAs single quantum well are presented. An error function profile is used to model the constituent atoms composition after interdiffusion. Results indicate that the exciton Stark shift in the disordered quantum well is greater than in the as-grown $10 \mathrm{~nm}$ wide $\mathrm{In}_{0.2} \mathrm{Ga}_{0.8} \mathrm{As}$ well, and that the change in electroabsorption near the fundamental exciton absorption peak is enhanced by $30 \%$ in the disordered quantum well for a $30 \mathrm{kV} / \mathrm{cm}$ electric field applied perpendicular to the well. These results may be used to achieve optical modulators with improved performance characteristics in strained quantum well structures. (C) 1995 American Institute of Physics.
\end{abstract}

In quantum well $(\mathrm{QW})$ structures excitonic effects produce large absorption peaks just below the interband transition energy, which become stable even at room temperature, and which sharpen the absorption edge. ${ }^{1}$ When an electric field is applied perpendicular to the QW layer, the exciton peaks remain resolved up to very high fields, and show significant shifts in the absorption edge. ${ }^{2}$ This quantumconfined Stark effect (QCSE) has been applied in QW optical modulators ${ }^{3}$ and in self-electro-optic-effect devices, ${ }^{4}$ where light propagates perpendicular to the QW layer. More recently, InGaAs/GaAs QW waveguide modulators, where light propagates parallel to the QW layer, have also been investigated. ${ }^{5}$ Such modulators are particularly interesting for monolithic integration with low threshold strained-layer laser diodes. ${ }^{6}$ For high device performance, such as high on/off ratio and a low drive voltage, it is desirable that a large Stark shift of the exciton is exhibited with applied electric field. A QW structure with a graded well compositional bottom has been proposed for this purpose. ${ }^{7}$

Disordering of QW structures involves the interdiffusion of constituent atoms across the well-barrier interfaces and results in a graded compositional profile. The extent of interdiffusion is a function of the disordering process parameters. ${ }^{8} \mathrm{QW}$ disordering leads to significant shifts in the absorption edge of QW structures, and is an attractive tool for band-gap engineering also in the context of monolithic integration. In this letter, the effects of QW disordering of a strained InGaAs/GaAs single QW on the Stark shift and electroabsorption are modeled to investigate the potential use

\footnotetext{
${ }^{a)}$ Present address: Department of Microelectronics, Universityo of Malta, Msida MSD06, Malta.

${ }^{b)}$ Present address: Department of Electrical and Electronic Engineering, The University of Hong Kong, Pokfulam Rd., Hong Kong.

${ }^{c}$ Present address: Department of Electronic and Electrical Engineering, University of Surrey, Guildford, Surrey GU2 5XH, United Kingdom.
}

of disordering of strained QWs for improved device performance.

In coherently strained pseudomorphic InGaAs/GaAs QWs a biaxial compressive strain perpendicular to the growth direction increases the energy gap between the conduction band and the degenerate valence bands, while a uniaxial compressive component of strain parallel to the growth direction results in heavy hole (hh)-light hole (lh) band edge splitting. ${ }^{9}$ In the model used here the electron and hole subbands are considered assuming the conduction band to be parabolic and that the lh subband shift due to the straininduced hh-lh band edge splitting is large enough to neglect valence band mixing. ${ }^{10,11}$ The disordering process is assumed to result in the interdiffusion of In and Ga atoms across the interface. ${ }^{12}$ An error function distribution is used to represent the compositional profile after disordering, ${ }^{13}$ and the extent of interdiffusion is characterized by the interdiffusion length $L_{d}=(D t)^{1 / 2}$, where $D$ is the diffusion coefficient and $t$ is the annealing time. The subbands are determined numerically following a scheme developed by Bloss ${ }^{14}$ for quasibound states, using spatially dependent effective masses and strain effects, ${ }^{15}$ and are then used to calculate the heavy-hole related $1 \mathrm{~S}$ exciton binding energies and wave functions by a perturbative-variational method. ${ }^{16}$ Numerical results are calculated for an as-grown QW, and for various values of interdiffusion length $L_{d}$. These results are next used to determine the room-temperature absorption coefficient near the fundamental absorption edge. Waveguide electroabsorption is considered here, with the light propagating along the plane of the QW layer. The absorption coefficient is calculated, for various values of $L_{d}$ and applied field, for the band edge $\Gamma$ region of the Brillouin zone taking into consideration only hh to conduction transitions, in view of the effects of the compressive strain on the lh subband, as discussed previously. The TE polarization absorption coefficient is determined by 


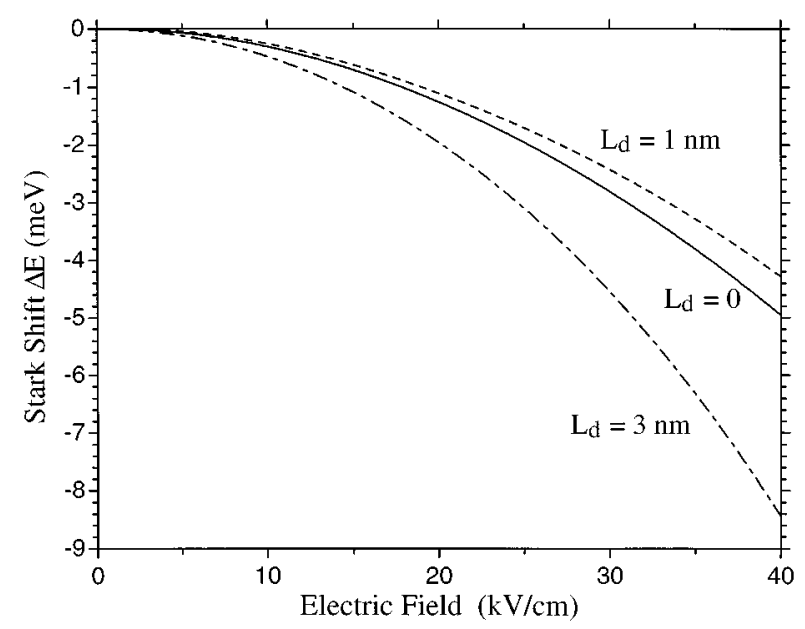

FIG. 1. Stark shift with applied electric field for an $\mathrm{In}_{0.2} \mathrm{Ga}_{0.8} \mathrm{As} / \mathrm{GaAs}$ single QW, with an as-grown $L_{z}=10 \mathrm{~nm}$, for various values of interdiffusion length $L_{d}$.

taking into consideration the $1 \mathrm{~S}$ exciton, and all the bound states, including the 2D enhancement Sommerfeld factor. Details of the calculations are given in Ref. 16. In this first analysis, the contributions from continuum states above the barrier gap have not been considered. This should only have a small effect on the electroabsorption spectrum near the fundamental absorption edge.

The as-grown QW structure considered here is a $10 \mathrm{~nm}$ InGaAs layer sandwiched between thick GaAs barriers. The effects of the applied field on the exciton Stark shift energy for the hh related transitions are shown in Fig. 1. In the initial stages of interdiffusion $\left(L_{d} \approx 1 \mathrm{~nm}\right)$ the Stark shift decreases slightly and then increases to a value larger than the as-grown case, showing a significantly larger value for $L_{d}=3 \mathrm{~nm}$. This reflects the changing carrier confinement profiles, as modified by disordering, strain, and applied field. For a given electric field the Stark shift depends on the width, depth, and shape of the confinement profile and is directly proportional to the carrier effective mass. The effective width of the tilted confinement profile, experienced by the electron and hh ground states under the action of the applied field, is larger in the disordered QW $\left(L_{d} \geqslant 2 \mathrm{~nm}\right.$, in this case) than in the as-grown QW. As a result, the applied field lowers the ground state subbands, and thus the ground state transition energy, to a greater extent in the disordered QW. In addition, the average of the spatially dependent effective masses over the well region is larger in the disordered $\mathrm{QW}$, as a result of the increasing effective masses with increasing distance from the well center. This heavier carrier mass results in a lower quantization level and thus greater sensitivity to the shape of the well, and therefore to the applied field. Thus, an enhanced Stark shift results in the disordered structure as compared to the as-grown structure. The increase in Stark shift in the disordered QW is similar in trend to results calculated for a graded-gap QW structure. ${ }^{17} \mathrm{It}$ can be seen that for $L_{d}=3 \mathrm{~nm}$, the Stark shift at $30 \mathrm{kV} / \mathrm{cm}$ is enhanced by a factor of $65 \%$ over the as-grown QW case. This enhancement could be the basis of improved device performance in electroabsorption modulators which can be

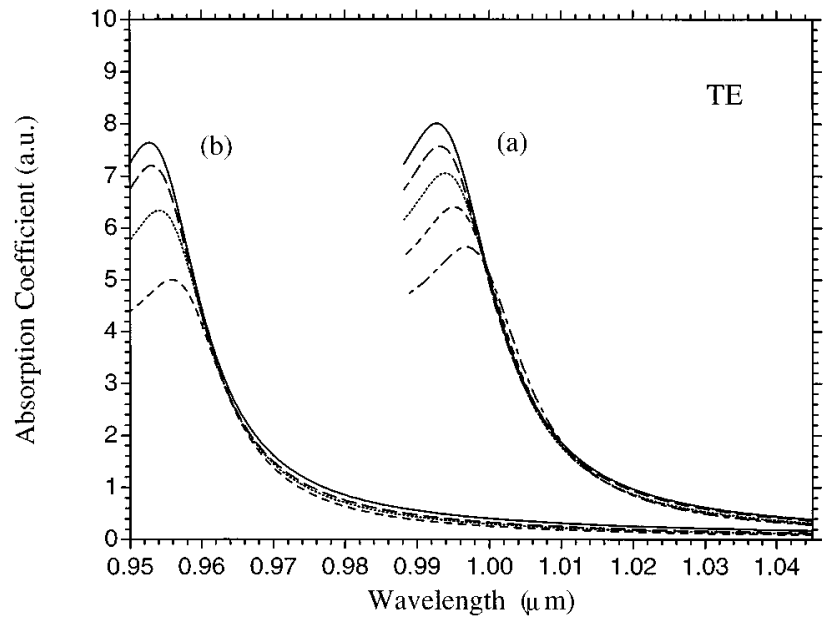

FIG. 2. Absorption coefficient wavelength spectra for TE polarization for an $\mathrm{In}_{0.2} \mathrm{Ga}_{0.8} \mathrm{As} / \mathrm{GaAs}$ single QW with $L_{z}=10 \mathrm{~nm}$, for various values of applied field: $F=0 \mathrm{kV} / \mathrm{cm}$ (solid), $10 \mathrm{kV} / \mathrm{cm}$ (long-dash), $20 \mathrm{kV} / \mathrm{cm}$ (dotted), 30 $\mathrm{kV} / \mathrm{cm}$ (short-dash), and $40 \mathrm{kV} / \mathrm{cm}$ (dot-dash), for (a) $L_{d}=0$, and (b) $L_{d}$ $=3 \mathrm{~nm}$.

driven with a low operating voltage and possess a high on/off ratio. The Stark shift values for the as-grown QW, compare favorably with calculated values reported for an InGaAs/ GaAs QW structure with similar barrier heights of 100 meV. ${ }^{10}$ The Stark shift values determined here for the strained, disordered InGaAs/GaAs QW are comparable to Stark shift values obtained when considering interdiffusion in an $\mathrm{Al}_{0.3} \mathrm{Ga}_{0.7} \mathrm{As} / \mathrm{GaAs} \mathrm{QW}$ with $L_{z}=10 \mathrm{~nm},{ }^{18}$ even though the conduction and hole barrier heights, for $L_{d}=0$ $\mathrm{nm}$, are 300 and $120 \mathrm{meV}$, respectively.

Figure 2 shows the TE polarized absorption coefficient spectra for an as-grown QW and for the disordered QW with $L_{d}=3 \mathrm{~nm}$, for various values of applied field. The exciton peak shifts to longer wavelengths with increasing applied field, reflecting the QCSE, at the same time decreasing in value. The applied field pulls apart the electron and hh wave functions thereby reducing the electron-hh wave function overlap, and thus the exciton peak. A similar trend in the behavior of the exciton peak at the fundamental absorption edge is observed in the disordered QW. The decrease in the exciton peak for similar values of applied field is larger for the disordered $\mathrm{QW}$ than for the as-grown $\mathrm{QW}$, since the electron-hh wave function overlap is reduced to a greater extent. In the disordered QW the electron and hh wave functions are less strongly confined and show a larger penetration out of the well, so that the two wave functions are pulled further apart by the electric field.

The change in the TE polarization absorption coefficient due to the application of an electric field, for wavelengths around the fundamental absorption edge, is shown in Fig. 3, for $L_{d}=0$ and $3 \mathrm{~nm}$, respectively. This change in electroabsorption is defined as $\Delta \alpha=\alpha(F)-\alpha(F=0)$. For the asgrown $\mathrm{QW}$ electric fields up to $40 \mathrm{kV} / \mathrm{cm}$ are considered, while for the disordered QW electric fields up to $30 \mathrm{kV} / \mathrm{cm}$ only are considered since tunneling out can be observed at $40 \mathrm{kV} / \mathrm{cm}$ for $L_{d}=3 \mathrm{~nm}$. It can be seen that a similar maximum value of $\Delta \alpha$ results near the respective fundamental 


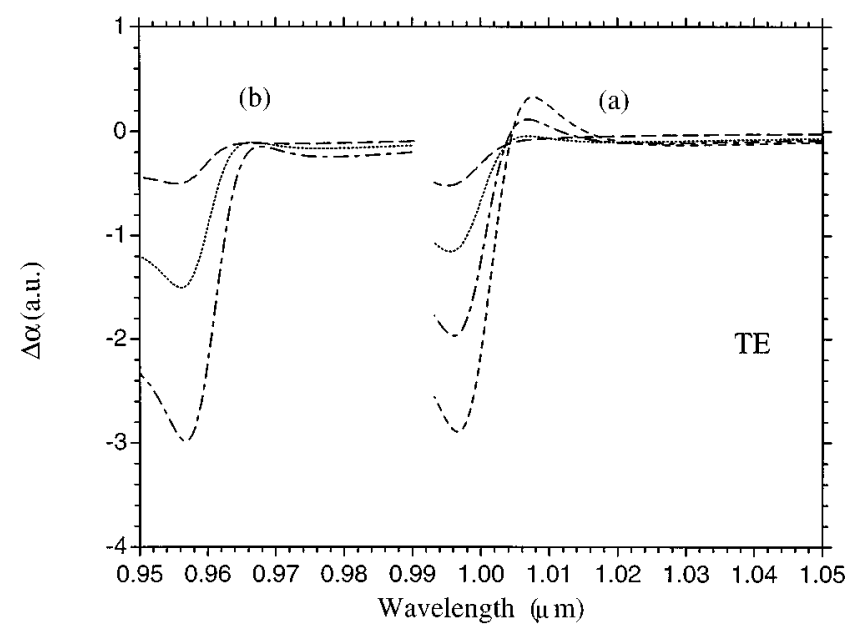

FIG. 3. Change in absorption coefficient for TE polarization $\Delta \alpha$, with applied field, for an $\mathrm{In}_{0.2} \mathrm{Ga}_{0.8} \mathrm{As} / \mathrm{GaAs}$ single QW with $L_{z}=10 \mathrm{~nm}$, where $\Delta \alpha=\alpha(F)-\alpha(F=0)$, for various values of applied field: $F=10 \mathrm{kV} / \mathrm{cm}$ (long-dash), $20 \mathrm{kV} / \mathrm{cm}$ (dotted), $30 \mathrm{kV} / \mathrm{cm}$ (dot-dash), and $40 \mathrm{kV} / \mathrm{cm}$ (shortdash), for (a) $L_{d}=0$, and (b) $L_{d}=3 \mathrm{~nm}$.

absorption edge in the two cases $L_{d}=0 \mathrm{~nm}$ with $F=40$ $\mathrm{kV} / \mathrm{cm}$, and $L_{d}=3 \mathrm{~nm}$ with $F=30 \mathrm{kV} / \mathrm{cm}$. This implies that the same maximum $\Delta \alpha$ can be achieved in the disordered structure at a lower operating voltage. This enhancement in electroabsorption can also be seen by comparing the maximum $\Delta \alpha$ for the as-grown and disordered $\mathrm{QW}$ at the same applied field. At $F=30 \mathrm{kV} / \mathrm{cm}$, the maximum $\Delta \alpha$ for the disordered QW is 30\% larger than that of the as-grown QW. Ideally this implies an improved on/off ratio for the same waveguide modulator length or a reduced device length for the same on/off ratio, the latter being particularly attractive in high speed optoelectronic circuit applications.

In conclusion, we have modeled the effects of disordering and strain on the quantum-confined Stark shift in an InGaAs/GaAs single QW. The results presented show that for a sufficiently large value of $L_{d}$ the exciton Stark shift in the disordered QW is greater than in the as-grown QW. For the QW considered here, the Stark shift is enhanced by about $65 \%$ for $L_{d}=3 \mathrm{~nm}$ at $F=30 \mathrm{kV} / \mathrm{cm}$. The corresponding change in maximum $\Delta \alpha$ when an electric field is applied for the disordered QW is $30 \%$ larger than that of the as-grown QW. This implies that for wavelengths around $1 \mu \mathrm{m}$, disordered, strained InGaAs/GaAs QWs could lead to an improved on/off ratio or reduced device length in waveguide modulators.

This work is supported in part by the HKU-CRCG Grant.

${ }^{1}$ S. Schmitt-Rink, D. S. Chemla, and D. A. B. Miller, Adv. Phys. 38, 89 (1989).

${ }^{2}$ J. S. Weiner, D. A. B. Miller, D. S. Chemla, T. C. Damen, C. A. Burrus, T. H. Wood, A. C. Gossard, and W. Wiegmann, Appl. Phys. Lett. 47, 1148 (1985).

${ }^{3}$ W. Dobbelaere, S. Kalem, D. Huang, M. S. Ünlü, and H. Morkoç, Electron. Lett. 24, 295 (1989).

${ }^{4}$ T. K. Woodward, T. Sizer, II, D. L. Sivco, and A. Y. Cho, Appl. Phys. Lett. 57, 548 (1990).

${ }^{5}$ O. Humbach, A. Stöhr, U. Auer, E. C. Larkins, J. D. Ralston, and D. Jäger, IEEE Photonics Technol. Lett. 5, 49 (1993).

${ }^{6}$ T. R. Chen, L. Eng, B. Zhao, Y. H. Zhuang, S. Sanders, H. Morkoç, and A. Yariv, IEEE J. Quantum Electron. QE-26, 1183 (1990).

${ }^{7}$ K. Nishi and T. Hiroshima, Appl. Phys. Lett. 51, 320 (1987).

${ }^{8}$ M. D. Camras, N. Holonyak, Jr., R. D. Burnham, W. Streifer, D. R. Scifres, T. L. Paoli, and C. Lindström, J. Appl. Phys. 54, 5637 (1983).

${ }^{9}$ G. C. Osbourn, J. Appl. Phys. 53, 1586 (1982).

${ }^{10}$ D. Coffey, J. Appl. Phys. 63, 4626 (1988).

${ }^{11}$ S. W. Corzine, R. H. Yan, and L. A. Coldren, Appl. Phys. Lett. 57, 2835 (1990).

${ }^{12}$ E. S. Koteles, B. Elman, C. A. Armiento, and P. Melman, Superlattices Microstruct. 9, 533 (1991).

${ }^{13}$ T. E. Schlesinger and T. F. Kuech, Appl. Phys. Lett. 49, 519 (1986).

${ }^{14}$ W. L. Bloss, J. Appl. Phys. 65, 4789 (1989).

${ }^{15}$ J. Micallef, E. H. Li, and B. L. Weiss, Superlattices Microstruct. 13, 125 (1993).

${ }^{16}$ J. Micallef, E. H. Li, and B. L. Weiss, Superlattices Microstruct. 13, 315 (1993).

${ }^{17}$ T. Hiroshima and K. Nishi, J. Appl. Phys. 62, 3360 (1987).

${ }^{18}$ E. H. Li, K. S. Chan, B. L. Weiss, and J. Micallef, Appl. Phys. Lett. 63, 533 (1993). 